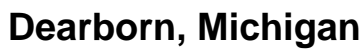 \\ NOISE-CON 2008 \\ 2008 July 28-31
}

\section{Vibration Response Models of a Stiffened Aluminum Plate Excited by a Shaker}

\author{
Randolph Cabell* \\ NASA Langley Research Center \\ Mail Stop 463 \\ Hampton, Va 23681
}

\begin{abstract}
Numerical models of structural-acoustic interactions are of interest to aircraft designers and the space program. This paper describes a comparison between two energy finite element codes, a statistical energy analysis code, a structural finite element code, and the experimentally measured response of a stiffened aluminum plate excited by a shaker. Different methods for modeling the stiffeners and the power input from the shaker are discussed. The results show that the energy codes (energy finite element and statistical energy analysis) accurately predicted the measured mean square velocity of the plate. In addition, predictions from an energy finite element code had the best spatial correlation with measured velocities. However, predictions from a considerably simpler, single subsystem, statistical energy analysis model also correlated well with the spatial velocity distribution. The results highlight a need for further work to understand the relationship between modeling assumptions and the prediction results.
\end{abstract}

\section{INTRODUCTION}

Accurate vibroacoustic models are necessary for improving the passenger experience in fixed-wing transports and rotorcraft. These models are also valuable for predicting vibroacoustic response to launch loads in spacecraft, both for analyzing human exposure and designing equipment to withstand the loads. At low frequencies, where structural wavelengths are long, finite element models perform well. At high frequencies, where modal densities are high in the structure and acoustic spaces, finite elements are not as useful [1, 2]. Issues with finite elements at high frequencies include prohibitively large mesh densities, uncertainty in the dynamic stiffness of joints, uncertainty in material thickness and damping, and uncertainty in external exciting forces. Modeling these uncertainties within finite elements requires considerable computational resources, even assuming the appropriate ranges and probability distributions of the uncertainties are known.

As a result of the limitations of deterministic models at high frequencies, Statistical Energy Analysis (SEA), which is based on a probabilistic approach, is often used on complex structures at high frequencies. SEA estimates frequency-averaged energy response functions of the subcomponents of the structure. This frequency averaging allows several simplifying assumptions to be made about the structural properties and the energy transmission between structural components [3]. A few overview reports and examples of SEA applied to aerospace structures are listed in the references [2, 4, 5]. Although SEA is widely used, it can be difficult to know when a given application

*randolph.h.cabell@ nasa.gov 
satisfies the assumptions required for SEA, and difficult to create an SEA model of a complex structure [6].

Recently, codes that implement a continuum form of SEA, known as Energy Finite Element Analysis (EFEA), have become commercially available. Whereas SEA assumes uniform energy distribution within a subsystem, EFEA assumes the energy flow at any point within a subsystem is proportional to the energy density [7, 8, 9, 2]. With this assumption, energy density can be described using a differential equation that resembles the equation for heat conduction, with appropriate boundary conditions applied at energy discontinuities in the structure [2]. This differential equation can be solved over a finite element grid using standard finite element techniques, which appeals to engineers already working with finite element models of a structure. While the validity of the EFEA approach has been questioned for two-dimensional structures [10, 11], it appears that the approach is valid under the same conditions as SEA (high modal density and reverberant field) [12].

The present work compares predictions from two EFEA codes, Comet EnFlow [13] and MESEFEA [14], the SEA code VA One [15], and the finite element code MSC.Nastran [16], with experimental measurements of the velocity response of a stiffened plate excited by a shaker.

The paper begins with a brief description of the test structure, followed by a discussion and underlying assumptions of finite element, SEA, and EFEA models. The experimental setup, shaker excitation and vibrometer response measurement are described next. Results are discussed, including the computed damping loss factors, the prediction of plate mean square velocities, and a metric to quantify the spatial accuracy of the velocity predictions from each model.

\section{TEST STRUCTURE}

The test structure consists of a stiffened aluminum plate, $1.27 \mathrm{~mm}$ thick, constructed to represent the sidewall of an aircraft fuselage but without curvature. The stiffened side of the plate is shown in fig. 1(a). Six stiffeners, or stringers, were attached to the plate in the $x$-direction, and three stiffeners, or frames, were attached in the $y$-direction. Both sets of stiffeners were attached along single rivet lines. Photos of the inverted hat-section stringer and frame are shown in Figs. 1(b) and 1(c), The flange of the Z-section was bolted to a flange of the stringer, as seen in fig. 1(b). The stringers were made from $1.02 \mathrm{~mm}$ thick aluminum; the frames were made from $1.27 \mathrm{~mm}$ aluminum. For the tests described here, the plate was clamped in the test window of a transmission loss suite such that the $y$-axis in fig. 1(a) was vertical. The outer $2.5 \mathrm{~cm}$ of the side edges of the plate were clamped, while the outer $1.27 \mathrm{~cm}$ of the top and bottom plate edges were clamped.

Four shaker excitation locations are also shown in fig. 1(a). More details on the excitation are given in the Experimental Setup section.

\section{NUMERICAL MODELS}

Finite element and energy models of the plate were developed for comparison with experimental data. A finite element mesh was used as input to the energy codes, so the development of the FE model is described first.

\section{A. Finite element}

Multiple finite element meshes of the test structure were created for different purposes as part of this study. A fine mesh with very small elements was created for a conventional frequency 


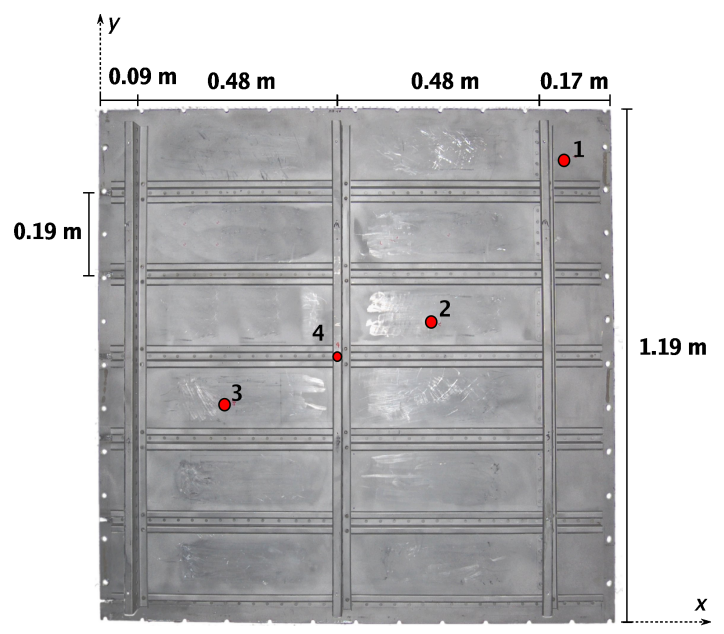

(a) Aluminum plate showing stringer frame, and shaker locations.

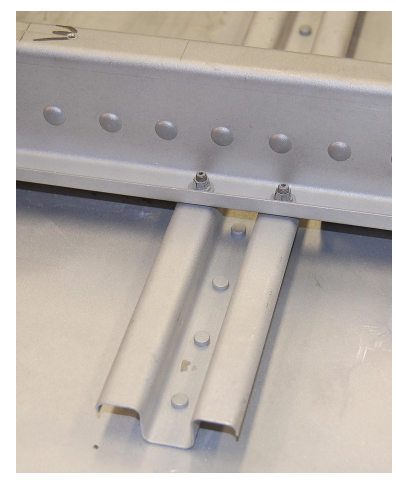

(b) Stringer and frame connection.

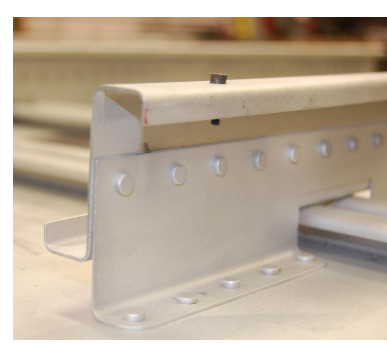

(c) Frame and shear tie.

Figure 1: Test structure.

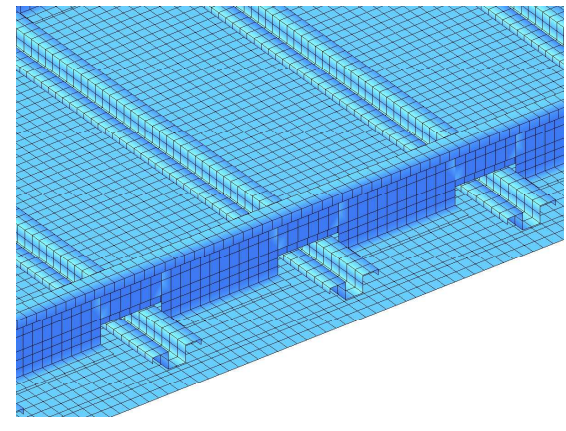

(a) Fine mesh for FE frequency response analysis.

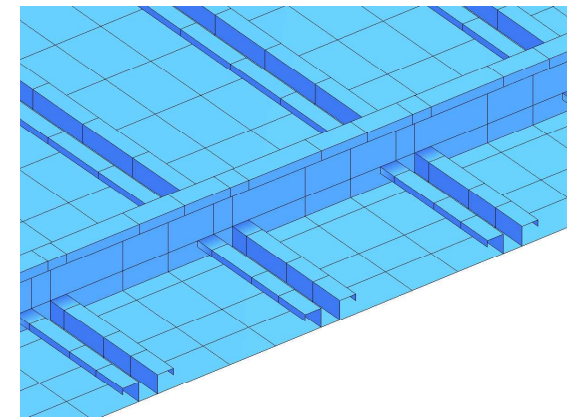

(b) Coarse mesh for export to MESEFEA code.

Figure 2: Finite element models showing plate elements for modeling stiffeners.

response analysis in MSC.Nastran [16], while coarser meshes were created as input to the energy finite element and statistical energy analysis codes.

The fine mesh consisted of plate elements (CQUAD4) to model both the aluminum plate and the stiffeners, following an approach described in an earlier analysis [17]. An isometric view of the meshed plate and stiffeners is shown in fig. 2(a). Riveted joints, where the stiffeners were attached to the plate and the shear ties were attached to the Z-section frame, were modeled using rigid body elements (RBE2s) coupling six degrees of freedom between neighboring nodes on the riveted components. Rigid body elements were also used to model the bolt attachment between the flanges on the frame and stringer. The mesh density for the plate elements was specified in order to provide at least six elements per flexural wavelength in the plate at approximately $2300 \mathrm{~Hz}$. The resulting mesh contained 113 elements in the $y$-direction and 117 elements in the $x$-direction, for a total of 13221 elements for the plate. A similar mesh density was used for the stiffeners to ensure compatible nodes along the rivet lines, resulting in 1738 plate elements for each frame and 1332 plate elements for each stringer. The total model contained 129629 degrees of freedom, and the 
total mass of the modeled plate was $8.96 \mathrm{~kg}$. Displacements of nodes along the outer $2.5 \mathrm{~cm}$ of the skin were constrained to zero to model the clamped boundary in the test fixture. Because multiple rows of nodes along the $x$ and $y$ plate edges were contained within the clamping fixture, setting their displacements to zero approximated the clamping effect of the fixture.

A direct frequency response solution was run in MSC.Nastran on the finite element model. A frequency dependent load representing the shaker force was applied at a single node at the shaker attachment point. Four separate frequency responses were computed, one for each shaker location. Each solution was computed at 20 logarithmically spaced frequencies within each onethird octave band from $100 \mathrm{~Hz}$ to $4000 \mathrm{~Hz}$. The resulting velocity predictions were averaged to obtain a single average velocity response for each one-third octave band. The frequency dependent input forces were obtained from an impedance head measurement in the experiment. Frequency dependent damping loss factors were applied in each one-third octave band. Computation of these loss factors is discussed in the results section.

Two coarse meshes of the structure were created for input to the energy codes. The first mesh, input to Comet EnFlow and VA One, used plate elements for the plate and one dimensional beam elements (CBEAM) for the stiffeners. The plate was modeled using a 20 by 24 grid of elements for a total of 480 plate elements. The total mass of the resulting plate model was $8.51 \mathrm{~kg}$. The second mesh, input to MES-EFEA, used plate elements for the plate and stiffeners, and a section of this mesh is shown in fig. 2(b). In this second mesh, areas where a stiffener overlapped the skin were modeled with plate elements of appropriate thickness (skin thickness plus stiffener thickness). The total mass of the resulting plate model was $8.82 \mathrm{~kg}$. The shear-tie cutouts at the frame-stringer intersections and the bolt attachments between the frame flange and the stringer were not modeled.

In the coarsely meshed models, the mesh only extended as far as the end of the stiffeners on the plate, leaving approximately $3 \mathrm{~cm}$ of each edge of the plate unmodeled. This was done to avoid creating an energy flow path along the unstiffened edge of the plate. Because this edge was clamped in the test fixture, such an energy flow path was unrealistic.

\section{B. Statistical Energy Analysis}

Subsystem partitionings were used to create two SEA models of the plate. A ribbed-panel model was created using VA One's ribbed-panel subsystem to model the entire stiffened plate assembly with a single SEA component. The ribbed-panel subsystem models a plate with up to two uniformly-spaced orthogonal sets of ribs, where each set of ribs is defined by its spacing, beam physical properties, and centroidal offset from the plate [18]. The total mass of the resulting plate model was $7.90 \mathrm{~kg}$. A second plate-beam SEA model was created that consisted of 24 plate subsystems, 3 beam subsystems for the frames, and 6 beam subsystems for the stringers. The VA One graphical representation of the plate-beam model is shown in fig. 3. The red lines in the figure denote line joints between the plates and beams, while the circles denote point joints between corners of neighboring plates. The model mass was $8.35 \mathrm{~kg}$.

Damping loss factors for all subsystems (plates, beams, and the ribbed shell) were assigned the same frequency dependent values. Computation of these loss factors is discussed in the results section. No energy was assumed to be lost through the clamped boundaries of the plate. Default coupling loss factors automatically computed by VA One were used for all junctions in the platebeam model.

Analytical coupling loss factors, such as the default values in VA One, generally assume the presence of a diffuse wave field in a subsystem, hence, the accuracy of the coupling factors depends 


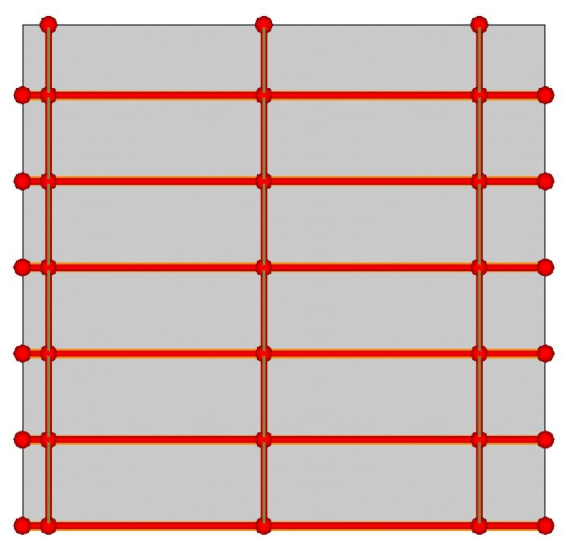

Figure 3: Plate-beam SEA model showing line and point junctions.

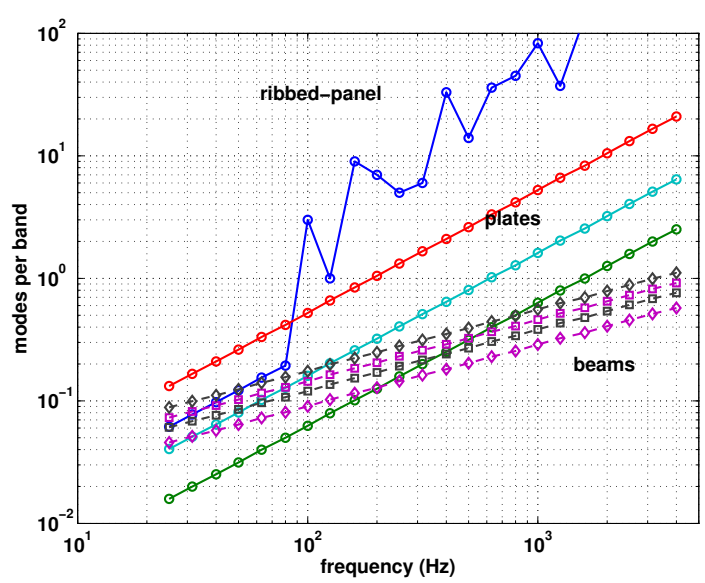

Figure 4: Modes per band in model subsystems.

on the validity of this assumption. A common guideline is to ensure each subsystem contains three or more natural frequencies in each analysis band. To check conformity with this guideline, the flexural modes per band for the plate and beam subsystems, computed by VA One, are shown in figure 4. The ribbed-panel subsystem, corresponding to the top line in the plot, contains more than three modes per band above the $160 \mathrm{~Hz}$ third octave band. However, none of the plate or beam subsystems contain three modes per band until much higher frequencies. These data suggest the plate-beam SEA model may not accurately represent energy flow in the structure at frequencies below several kilohertz.

\section{Energy Finite Element Models}

The two EFEA codes offered three ways to model the plate stiffeners: (1) using one-dimensional beam elements with their associated degrees of freedom included in the model; (2) using onedimensional beam elements with their degrees of freedom not included in the model, but the beam properties are used to compute power transmission coefficients between neighboring plates; and (3) using plate elements to create detailed models of the beams. The first method was used for the Comet EnFlow model, while the third method was used for the MES-EFEA model. An isometric view of a portion of the mesh used in the MES-EFEA model is shown in fig. 2(b), The MES-EFEA model contained 1704 plate elements, while the EnFlow model contained 480 plate elements and 192 beam elements.

Preprocessors for the two EFEA codes read in a finite element mesh and detect energy discontinuities due to geometric features, changes in material properties, and intersections between components. The preprocessors disconnect the finite element model at these discontinuities, add nodes, and update the connectivity matrix. For example, the EnFlow preprocessor detected energy discontinuities where the beam elements were attached to plate elements. The MES-EFEA preprocessor detected many more discontinuities due to the modeling approached used in its finite element mesh. For example, discontinuities were detected where the stiffeners were attached to the plate, but also within the stiffeners themselves, wherever $90^{\circ}$ bends occurred. At energy discontinuities, the EFEA codes apply boundary conditions to capture energy transmission and reflection through the joint. As in SEA, the boundary conditions assume a diffuse wave field in 
each structural component at the joint. Because the MES-EFEA model subdivided the structure into even smaller components than the plate-beam SEA model, the assumption of a diffuse wave field in these components seems invalid over much of the frequency range in fig. 4. As with the plate-beam SEA model, this suggests the energy transmission may be poorly modeled by the MES-EFEA model.

All materials within the EFEA models were assigned the same frequency averaged damping loss factors. Computation of these loss factors is discussed in the results section. No energy was assumed to be lost through the clamped boundaries of the plate.

It should be noted that the EFEA models were constructed with assistance and feedback from the developers of the two EFEA codes. No such assistance was received for the SEA models.

\section{EXPERIMENTAL SETUP}

The test structure was mounted vertically in the test window of the Structural Acoustic Loads and Transmission (SALT) facility at NASA Langley. This facility consists of a $278 \mathrm{~m}^{3}$ reverberant room and a $337 \mathrm{~m}^{3}$ anechoic room connected by a $1.2 \mathrm{~m}$ square window. The outer $2.54 \mathrm{~cm}$ of the left and right edges of the plate were clamped in the test fixture, as were the outer $1.27 \mathrm{~cm}$ of the top and bottom plate edges. The stiffened side of the plate faced the reverberant room, and a single shaker was mounted to this side. Four excitation locations, indicated by the numbered circles in fig. 1(a), were studied. The first three locations were slightly offset from centers of subpanels, while location 4 was on the middle frame stiffener. In all cases, the applied force was normal to the plane of the plate. An impedance head was attached between the shaker and the plate to measure the input force and drive point acceleration. The shaker was driven by a pseudo-random signal with a bandwidth of 50 to $5000 \mathrm{~Hz}$.

To measure out-of-plane plate velocity, a scanning laser vibrometer was positioned in the receiver chamber of the transmission loss suite. The velocity was measured over a uniformly-spaced grid with $47 \times 47$ measurement points, for a total of 2209 points, with approximately $2.5 \mathrm{~cm}$ between measurement points. The plate's surface was coated with glass beads to increase the diffuse reflectivity of the bare aluminum. The beads and associated adhesive added $0.26 \mathrm{~kg}$ to the bare structure mass of $8.74 \mathrm{~kg}$ (approximately $3 \%$ ), for a total mass of $9.00 \mathrm{~kg}$.

\section{RESULTS}

The finite element model and laser vibrometer produced a large amount of data that required considerable post-processing before comparison with the energy models. Post-processing tasks included translating results into a common coordinate system, ensuring consistent units, and converting to third-octave bands. Due to the space limitations of this paper, only results from shaker excitation points 2 and 4 (fig. 1(a) are discussed here. The entire data set and finite element model are publicly available for further studies [19].

The finely-meshed finite element model used for the frequency response analysis was not updated to match the experimental data. However, the first three predicted natural frequencies at 53, 100 , and $119 \mathrm{~Hz}$ were were within $\pm 10 \%$ of experimentally measured natural frequencies.

Different approaches were used to represent the shaker excitation. For the finite element frequency response analysis, both shaker locations were modeled as a force applied to a single node using experimentally measured narrowband force values. For the energy models, shaker location

2 was modeled as a flexural power input to the plate using experimentally measured power values 


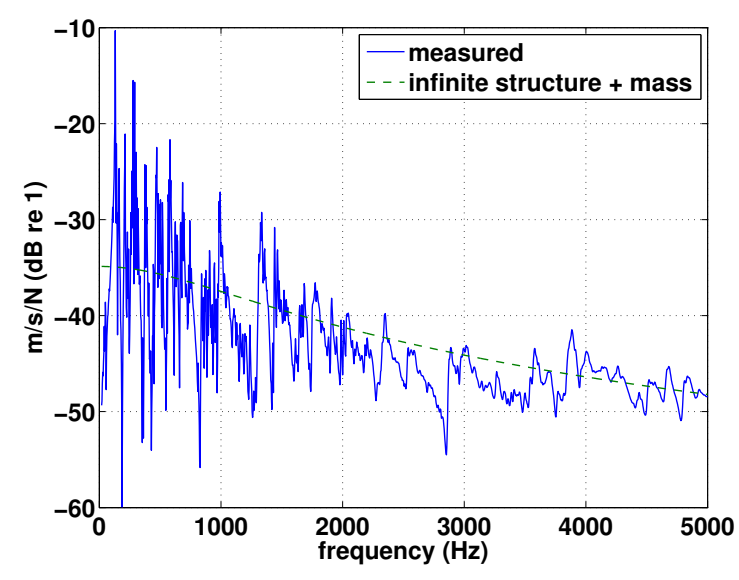

Figure 5: Drive point mobility at shaker location 2 .

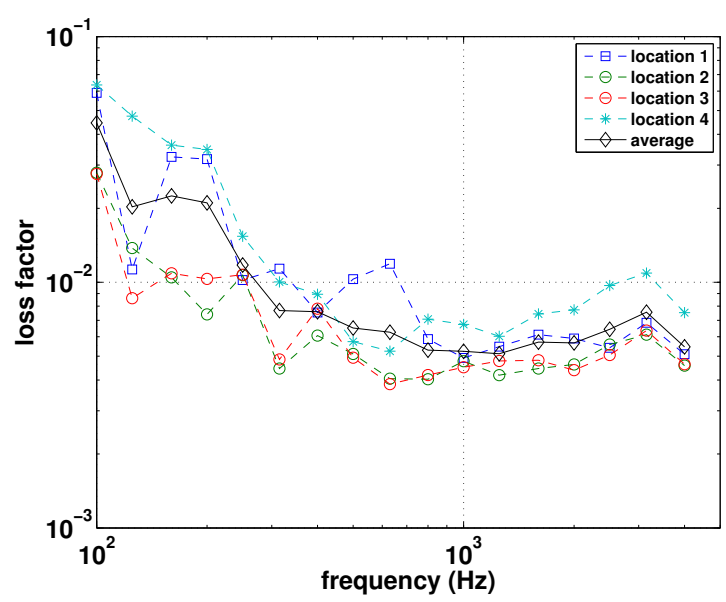

Figure 6: Loss factors computed using power injection method.

in one-third octave bands. Shaker location 4 was more complicated, but was again modeled as a flexural power input to the ribbed-panel SEA model. In the plate-beam SEA model, shaker 4 was modeled as a power input to the beam subsystem representing the middle frame stiffener. In EnFlow, the input power for shaker 4 was quartered and applied to the four corner nodes of the four plate elements that joined directly beneath the excitation location. In MES-EFEA, the power was also quartered, but was then applied to the four vertices of the CQUAD4 element on the top flange of the frame nearest the shaker location.

\section{A. Drive Point Mobility}

The drive point mobility at shaker location 2 is shown in fig. 5. The solid blue curve represents the magnitude of the measured mobility while the dashed green curve is the mobility of an infinite aluminum plate $1.27 \mathrm{~mm}$ thick, with an $8 \mathrm{~g}$ mass in series with the plate. The mass is necessary to account for the impedance of the end mass of the impedance head and the nut used to attach the impedance head to the plate. The force values input to the Nastran model were adjusted to account for the presence of the $8 \mathrm{~g}$ mass.

\section{B. Damping Loss Factors}

Frequency-averaged damping loss factors were determined using a power injection method described in [20]. Rather than estimate loss factors for individual components of the structure, an average loss factor for the entire structure was computed from the experimental data. This average loss factor accounted for all energy losses from the structure, such as material damping, joint losses, losses at the clamped boundary, and acoustic radiation losses. The loss factor, $\eta(\omega)$, was computed as

$$
\eta(\omega)=\frac{\Delta E}{E_{S E}} \approx \frac{E_{\text {input }}}{E_{K E}} \approx \frac{\operatorname{Re}\left[h_{f v}(\omega)\right]}{\omega m \sum_{i=1}^{N}\left|h_{i f}\right|^{2}}
$$

where $\Delta E$ is the dissipated energy, $E_{S E}$ is the strain energy, $E_{\text {input }}$ is the input energy, and $E_{K E}$ is the total kinetic energy. The rightmost expression in Eq. 1 shows how the energy quantities were computed from experimental data, where $h_{f v}$ is the drive point mobility, and $h_{i f}$ is the transfer 


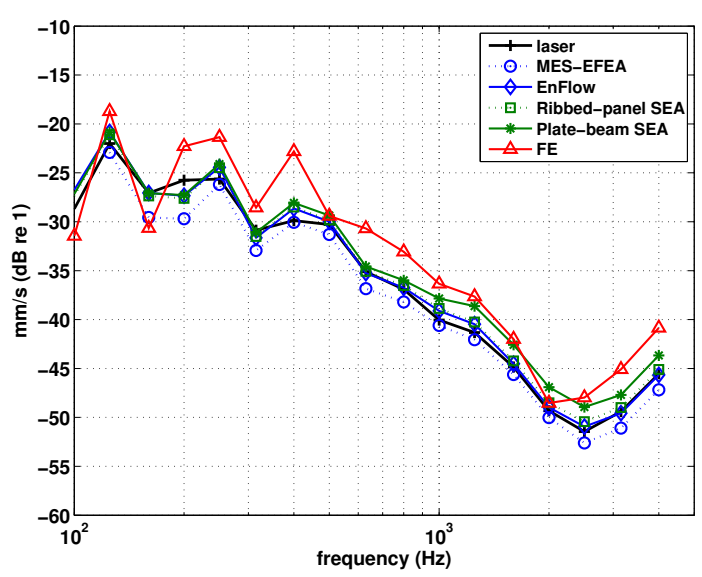

(a) Shaker location 2.

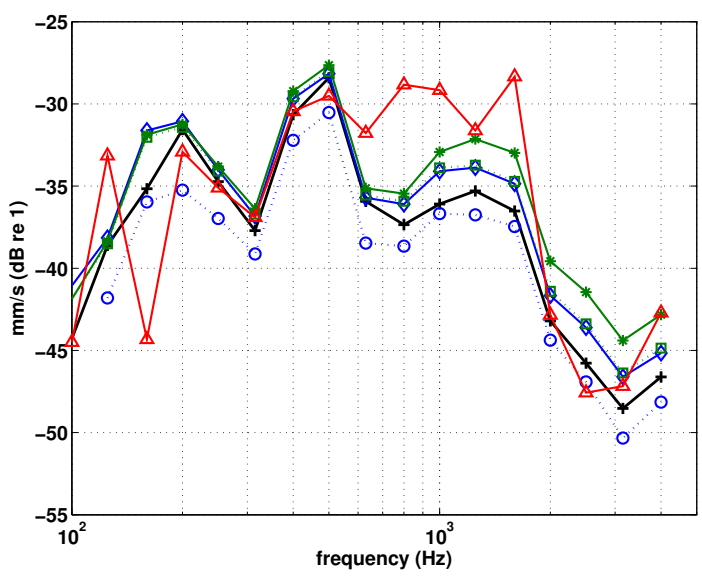

(b) Shaker location 4 (legend as in fig.7(a)).

Figure 7: Average plate velocity: models and measurement.

mobility, measured at $N$ uniformly spaced points on the plate. The mass of the structure is assumed to be uniformly distributed, and $m$ in Eq. 1] denotes the portion of the total mass assigned to an individual laser measurement point. The total mass used to compute $m$ was the mass corresponding to the plate-beam SEA model, or $8.35 \mathrm{~kg}$.

Computed loss factors at one-third octave band center frequencies for individual shaker locations and an average over the four locations are shown in fig. 6. Location 4 generally had the highest loss factor, which may reflect energy losses through the riveted joints connecting the shear-tie to the Z-section and plate.

\section{Average plate velocity}

Predictions of the spatially averaged root-mean-square velocity of the plate are compared with measured values in fig. 7 for shaker locations 2 and 4 . The measured values are obscured in fig.7(a) due to the good agreement between the models and measurement. At location 2, the energy methods are within $\pm 3 \mathrm{~dB}$ of measurements across the analysis bandwidth, and $\pm 4 \mathrm{~dB}$ at location 4. It should be re-emphasized that the models used experimentally measured power values and experimentally computed damping loss factors. Hence, as long as each model accurately captured the mass of the system, the root-mean-square velocity prediction should be accurate. The finite element results did not agree as closely with the measurements, particularly at shaker location 4. This could be explained by (1) the finite element model was never updated to match experimental natural frequencies, and (2) the finite element model used measured forces instead of measured powers. The drive point mobility has to be accurate in the FE model in order for the input power to be accurate. This is not so difficult at shaker location 2 at high frequencies, since the mobility approaches an infinite plate mobility, but it is more difficult at location 4 on the stiffener. In addition, the power from location 4 was coupled to the skin by two riveted joints (the shear tie to the Z-section, and the shear tie to the skin), and these joints may not have been well-modeled by the rigid body elements in the finite element model. 


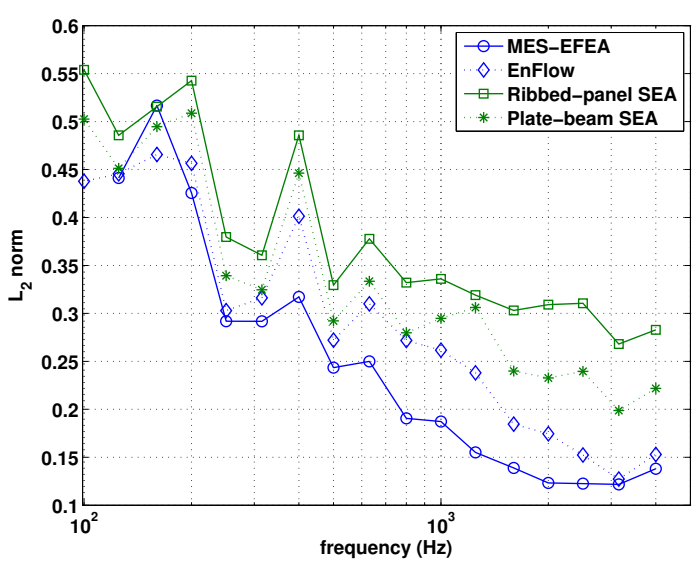

(a) $L_{2}$ at shaker location 2 .

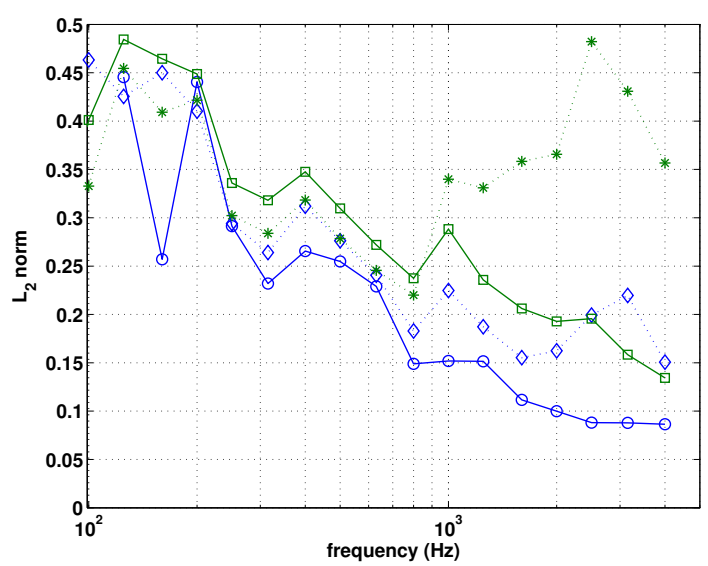

(b) $L_{2}$ at shaker location 4 .

Figure 8: Spatial accuracy of velocity predictions (lower is better).

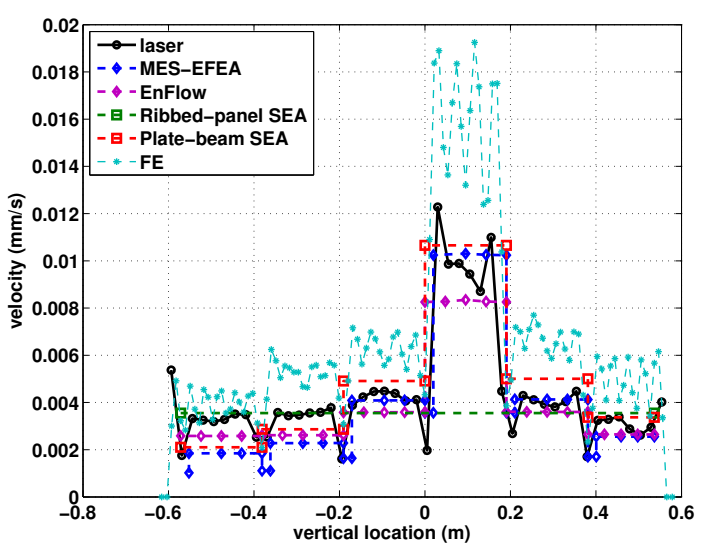

(a) Velocity in $y$-direction, shaker location 2.

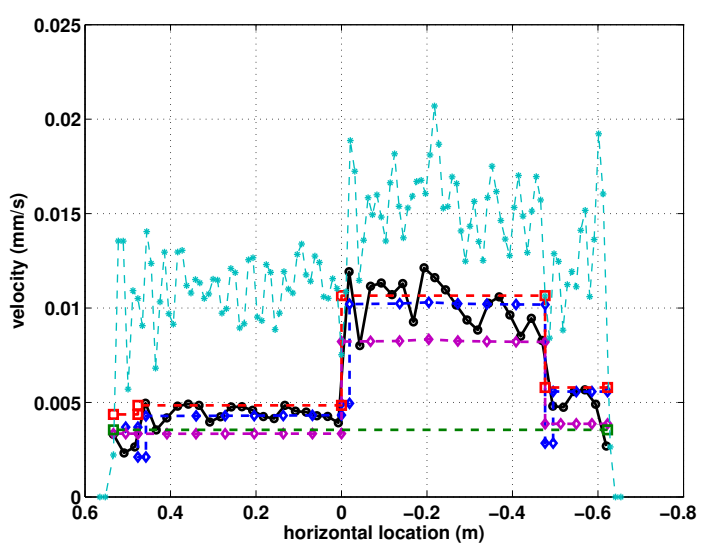

(b) Velocity in $x$-direction, shaker location 2.

Figure 9: Spatial velocity variation for shaker location 2 at $3150 \mathrm{~Hz}$.

\section{Spatial Variation of Plate Velocity}

The ability of the models to describe the spatial variation of velocity across the entire test structure was quantified using the metric

$$
L_{2}=\frac{\left\langle(\hat{v}-v)^{2}\right\rangle}{\left\langle v^{2}\right\rangle}
$$

where $\left\langle v^{2}\right\rangle$ is the mean square plate velocity, and $\left\langle(\hat{v}-v)^{2}\right\rangle$ is the mean square of the point by point difference between the predicted and measured plate velocities. If the predicted velocity is zero, $L_{2}=1$, whereas $L_{2}=0$ if $\hat{v}=v$. To compute the point by point difference between predictions and measurement, the predicted velocities were mapped onto a grid compatible with the laser measurement grid.

$L_{2}$ values for the energy models are shown in fig. 8 for shaker locations 2 and 4 . At shaker location 2, the $L_{2}$ values generally trend downward with frequency, indicating better spatial correlation between models and measurement with increasing frequency. The ribbed-panel SEA model, which predicted uniform velocity across the entire plate, also follows this decreasing trend in $L_{2}$ with fre- 


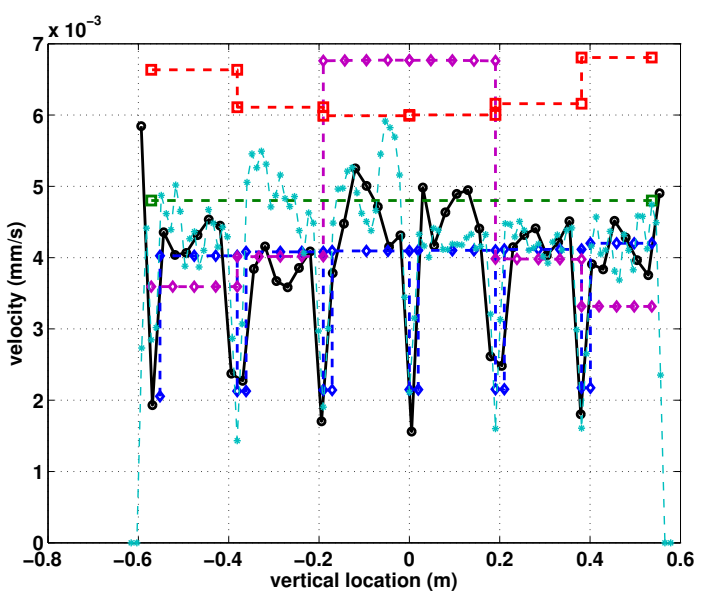

(a) Velocity in $y$-direction, shaker location 4.

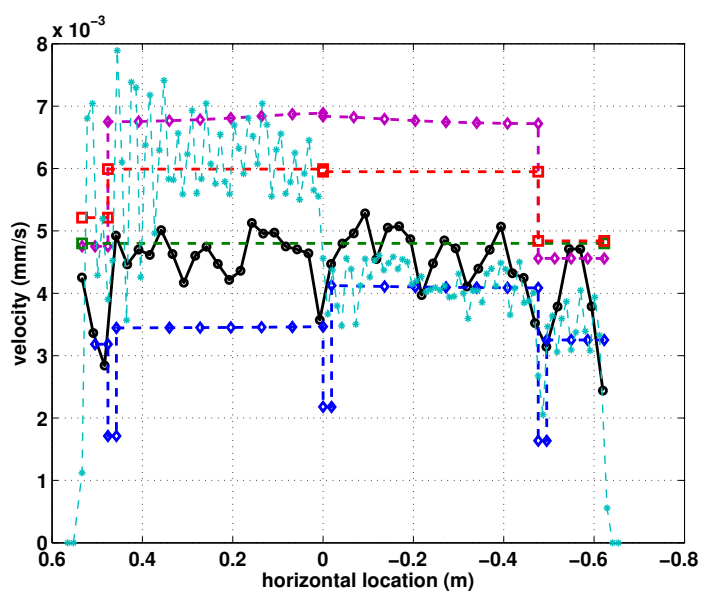

(b) Velocity in $x$-direction, shaker location 4.

Figure 10: Spatial velocity variation for shaker location 4 at $3150 \mathrm{~Hz}$.

quency. This suggests the trend is partly due to a velocity that is increasingly uniform across the plate at higher frequencies. Nonetheless, the lower $L_{2}$ values for the EFEA and plate-beam SEA models relative to the ribbed-panel SEA model in Fig. 8(a) show there is some improvement in spatial accuracy provided by these models.

At shaker location 4, the benefit of the EFEA and plate-beam SEA models relative to the ribbed-panel model is not as consistent. Above $1000 \mathrm{~Hz}$, the plate-beam SEA model shows worse spatial correlation than any other model. This could be due to either a poor representation of the power input or inaccurate coupling loss factors. Because this discrepancy occurs at higher frequencies where modal density is high and coupling loss factors are more accurate, it is reasonable to conclude a poor model of the power input is responsible. Similar reasoning could be used to explain the increased error of the EnFlow model above $2000 \mathrm{~Hz}$.

More detail on the spatial velocity distribution is given in Figs. 9 and 10 , which show velocities in vertical $(y)$ and horizontal $(x)$ slices through the plate in the $3150 \mathrm{~Hz}$ third-octave band. These slices intersect the subpanel containing shaker location 2 (see Fig. 1(a). In figs. 9(a) and 10(a), a vertical location of $-0.6 \mathrm{~m}$ corresponds to the bottom of the plate; in figs. 9 (b) and 10 (b), a horizontal location of 0.6 corresponds to the left side of the plate in fig. 1(a). To reduce variance, the laser and finite element velocities in the plots at a given $y$ coordinate were averaged over the $x$ coordinate up to the edges of a subpanel. A similar treatment was done for data in the horizontal direction.

Velocity variations from the driven subpanel to neighboring subpanels are most evident in the plots for shaker location 2 (fig. 9). The $x$-direction data in fig. 9(b) suggests the FE model did not capture the frame stiffness as accurately as the energy models.

Variations between the models are more evident in the data for shaker location 4, in fig. 10. The data in the plot shows that the plate-beam SEA model overpredicted the response of the top and bottom subpanels relative to the measured data. EnFlow overpredicted the response of the subpanels where the shaker power was applied in that model, relative to the measured data. As was noted with the $L_{2}$ data, these discrepancies are most likely due to a poor representation of the shaker input power in these two models.

It should be noted that the spatial accuracy of the FE model was exceptionally good in this 
third-octave band, and was much worse (generally exceeding the measured velocities) in nearly all other frequency bands.

\section{E. Limitations of the comparative study}

The study described in this paper has several limitations worth stating: (1) the available test structure was small and lightly damped, with many paths for reverberant energy to be distributed around the structure. As a result, the velocity was relatively uniform across the structure and was forgiving of modeling errors. (2) The measurements were from a single experiment in a single facility, and don't capture important response variability due to manufacturing and facility variations. In particular, the $L_{2}$ and velocity slice plots might look different if measured on a second stiffened plate structure, and lead to different conclusions about which code was most accurate. (3) The modeling results reflect the experience, judgment, and errors of a single person as much as they reflect the capabilities of the different codes. To counteract this factor, the experimental data and finite element model are available to the public [19] for further study. (4) The EFEA codes use a preprocessor to detect energy discontinuities in an imported finite element mesh, but the accuracy of this preprocessor was not studied. The accuracy depends on how the discontinuities are represented in the finite element mesh and on the analysis bandwidth, since the importance of a discontinuity can vary with wavelength. (5) The EFEA and plate-beam SEA models contain subsystems, or, structural components between energy discontinuities, that have very few modes per band in much of the analysis bandwidth studied here (see fig. 4). However, the default coupling loss factors provided by all of the codes assume a diffuse field, or high modal density, in components that meet at a joint. In spite of this apparent violation of a fundamental assumption of the nature of power transmission across a joint, the predictions correlated well with measurements.

\section{CONCLUSIONS}

Finite element, energy finite element, and statistical energy analysis predictions of the velocity response of a stiffened plate excited by a shaker were compared with velocities measured by a laser velocimeter. For shaker excitation of a subpanel, the average predicted plate velocity from the energy models agreed with experimental data to within $\pm 3 d B$ from $100 \mathrm{~Hz}$ to $4000 \mathrm{~Hz}$. Slightly greater variation was seen when the shaker excited a stiffener of the plate, but the energy models still agreed with measurements within $\pm 4 d B$ across the analysis bandwidth. This good agreement is largely due to the use of experimentally measured input powers and experimentally measured damping loss factors in the energy models. The energy finite element models captured the spatial velocity variation slightly better than a finely-partitioned SEA model when the shaker was attached to a subpanel. When the shaker was attached to a frame stiffener, the velocity distribution was relatively uniform across the plate. Greater variability among the models was seen in this case, which was attributed to differences in how the shaker input power was represented in the models. An energy finite element model again showed the highest correlation with the measured data for this shaker case. Further work is needed to understand the relatively good performance of the energy finite element models in spite of apparently violating a diffuse field assumption at energy discontinuities. 


\section{REFERENCES}

[1] Richard H. Lyon and Richard G. DeJong. Theory and Application of Statistical Energy Analysis. ButterworthHeinemann, second edition, 1995.

[2] R.S. Langley and F.J. Fahy. High-frequency structural vibration. In Advanced Applications in Acoustics, Noise and Vibration, Eds. Frank Fahy and John Walker, pages 490-529. Spon Press, 2004.

[3] Frank J. Fahy. Statistical energy analysis: a critical overview. Philosophical Transactions of the Royal Society of London, 346:431-447, 1994.

[4] J.S. Alex Lin. Airplane interior noise modeling using statistical energy analysis approach. In 5th AIAA/CEAS Aeroacoustics Conference, AIAA-99-1903, Bellevue, WA, May 1999.

[5] C.A. Yoerkie, J.A. Moore, and J.E. Manning. Development of rotorcraft interior noise control concepts: Phase 1: Definition study. Technical Report N83-30166, NASA, Hampton, VA, May 1983.

[6] G. Maidanik. Some elements in statistical energy analysis. Journal of Sound and Vibration, 52(2):171-191, 1977.

[7] O.M. Bouthier and R.J. Bernhard. Simple models of the energetics of transversely vibrating plates. Journal of Sound and Vibration, 182(1):149-166, 1995.

[8] M.J. Smith. A hybrid energy method for predicting high frequency vibrational response of point-loaded plates. Journal of Sound and Vibration, 202(3):375-394, 1997.

[9] Weiguo Zhang, Aimin Wang, and Nickolas Vlahopoulos. An alternative energy finite element formulation based on incoherent orthogonal waves and its validation for marine structures. Finite Elements in Analysis and Design, 38:1095-1113, 2002.

[10] R.S. Langley. On the vibrational conductivity approach to high frequency dynamics for two-dimensional structural components. Journal of Sound and Vibration, 182(4):637-657, 1995.

[11] A. Carcaterra and A. Sestieri. Energy density equations and power flow in structures. Journal of Sound and Vibration, 188(2):269-282, 1995.

[12] A. Sestieri and A. Carcaterra. Space average and wave interference in vibration conductivity. Journal of Sound and Vibration, 263:475-491, 2002.

[13] Comet Technology Corporation. Comet EnFlow User's Manual, 2007.

[14] Michigan Engineering Services LLC. MES/EFEA 2.0: User's Manual, 2007.

[15] ESI Group. VA One 2006 User's Guide, 2006.

[16] MSC.Software Corporation, Santa Ana, CA. MSC Nastran 2007 rl: Release Guide, 2007.

[17] Ralph D. Buehrle, Gary A. Fleming, Richard S. Pappa, and Ferdinand W. Grosveld. Finite element model development for aircraft fuselage structures. In XVIII International Modal Analysis Conference, San Antonio, TX, Feb, 2000, February 2000.

[18] P.G. Bremner. Vibro-acoustics of ribbed structures - a compact modal formulation for SEA models. In Noise-Con 94, pages 545-550, Ft. Lauderdale, Florida, May 1994.

[19] Randolph H. Cabell, Jake Klos, Ralph Buehrle, and Noah Schiller. Vibroacoustic response data of stiffened panels and cylinders. In Noise-Con 2008, Dearborn, Michigan, July 2008.

[20] Brandon C. Bloss and Mohan D. Rao. Estimation of frequency-averaged loss factors by the power injection and the impulse response decay methods. Journal of the Acoustical Society of America, 117(1):240-249, January 2005. 